\title{
Le prophète des Terjuševo
}

Un mouvement de paysans mordves au début du XIX ${ }^{\mathrm{e}}$ siècle

The Teryushevo prophet: a movement of Mordvin peasants at the beginning of the 19th century

ТЕРЮШЕВСКИЙ ПРОРОК : ДВИЖЕНИЕ МОРДОВСКИХ КРЕСТЬЯН В НАЧАЛЕ 19. ВЕКА

\section{Vladimir Kuz'mič Abramov}

Traducteur : Antoine Chalvin

\section{OpenEdition}

\section{Journals}

Édition électronique

URL : https://journals.openedition.org/efo/5132

DOI : 10.4000/efo.5132

ISSN : 2275-1947

Éditeur

INALCO

Édition imprimée

ISBN : 978-2-343-08571-5

ISSN : 0071-2051

\section{Référence électronique}

Vladimir Kuz'mič Abramov, «Le prophète des Terjuševo », Études finno-ougriennes [En ligne], 47 | 2015, mis en ligne le 13 juillet 2016, consulté le 20 septembre 2021. URL : http://journals.openedition.org/ efo/5132 ; DOI : https://doi.org/10.4000/efo.5132

Ce document a été généré automatiquement le 20 septembre 2021.

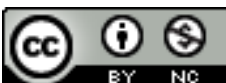

Études finno-ougriennes est mis à disposition selon les termes de la Licence Creative Commons Attribution - Pas d'Utilisation Commerciale 4.0 International. 


\section{Le prophète des Terjuševo}

Un mouvement de paysans mordves au début du XIX siècle

The Teryushevo prophet: a movement of Mordvin peasants at the beginning of the 19th century

ТЕРЮШЕВСКИЙ ПРОРОК : ДВИЖЕНИЕ МОРДОВСКИХ КРЕСТЬЯН В НАЧАЛЕ 19. ВЕКА

\section{Vladimir Kuz'mič Abramov}

Traduction : Antoine Chalvin

1 Le $\mathrm{XIX}^{\mathrm{e}}$ siècle arriva en Europe sur les ailes de la Révolution française. La Déclaration des droits de l'homme et du citoyen, adoptée en 1789 par l'Assemblée constituante, proclama le droit à la liberté individuelle, à la liberté de parole, le droit de réunion, de religion, le droit à l'égalité des citoyens devant la loi, ainsi que le droit de résister à l'oppression, présentés comme des droits imprescriptibles. Le servage fut aboli, les paysans reçurent des terres et devinrent des propriétaires libres. Les appels à l'égalité, à la liberté et à la souveraineté populaire se propagèrent dans les pays voisins et pénétrèrent jusque dans la lointaine Russie, agitant les esprits et préparant l'opinion publique à comprendre que le servage avait fait son temps. Effrayés, les seigneurs féodaux déclarèrent la guerre à la France afin d'écraser la révolution par les armes. Ils furent rejoints dans leur combat par les autocrates russes : Catherine, son fils Paul, puis son petit-fils Alexandre, qui devint tsar en 1801.

2 Une réaction originale à la Révolution française fut, dans les années 1804-1810, le mouvement national et religieux des paysans mordves du volost' de Terjuševo (province de Nijni-Novgorod, dans la région de la Volga). Aucun autre volost' de cette province, depuis le milieu du $\mathrm{xvI}^{\mathrm{e}}$ siècle, n'avait sans doute causé aux propriétaires fonciers autant d'ennuis et de terreur que celui-ci. Sa population mordve, compacte, avait préservé pendant des siècles ses traditions et ses rites religieux et avait gardé sa dignité dans ses relations avec la noblesse. Dès le début du xvII siècle, elle vivait dans le servage; elle fut en majeure partie baptisée à partir du milieu du xvIII siècle. Cependant, malgré tous les efforts et toutes les ruses des propriétaires, des fonctionnaires et du clergé, elle avait toujours conservé son esprit indépendant et fier, son identité et son désir de liberté. 
Une partie importante des paysans du volost' appartenait alors au prince Egor Gruzinskij (descendant russifié du roi Arčil d'Iméréthie ${ }^{1}$, qui s'était réfugié en Russie au $\mathrm{XVII}^{\mathrm{e}}$ siècle, fuyant les Turcs et les Perses). Quand la fille du propriétaire épousa le comte de Saint-Priest (un Français émigré en Russie après la révolution de 1789), le domaine de Terjuševo fut attribué à celui-ci à titre de dot. Chassé par les paysans français, le comte devint propriétaire de paysans mordves. Les raisons qui avaient poussé leur nouveau maître à venir en Russie étaient sans aucun doute bien connues des habitants du volost' et alimentaient leurs conversations. L'émancipation des serfs dans la lointaine France donnait quelque espoir qu'une telle libération pourrait se produire aussi en Russie. Les rumeurs sur la préparation d'un tel projet, la dégradation de la situation des paysans du fait des guerres incessantes menées par l'Empire, ainsi que la gestion calamiteuse du domaine par Egor Gruzinskij, poussèrent les Mordves de Terjuševo à la révolte.

4 À l'été et à l'automne 1804, des rebelles pillèrent l'administration seigneuriale, détruisirent les actes et documents relatifs au servage qui s'y trouvaient, cessèrent d'exécuter la corvée et s'emparèrent collectivement des récoltes du domaine. L'intendant, qui essaya de leur résister, fut tué. Des unités de l'armée régulière furent envoyées sur les lieux. Les habitants de Terjuševo, comme toujours, s'organisèrent pour accueillir l'expédition punitive et lui résistèrent. Bien sûr, les soldats qui disposaient de fusils remportèrent la victoire sur les paysans, qui étaient armés seulement de fourches et de haches, mais les paysans avaient connu l'espoir de la libération et cet espoir ne s'était pas éteint. Ils n'allèrent pas accomplir la corvée, firent des coupes pour leur propre compte dans les bois du domaine, fauchèrent les prairies du domaine, et se rassemblèrent à l'occasion de cérémonies religieuses traditionnelles. La dimension religieuse a toujours été présente, à un degré ou à un autre, dans les révoltes de Terjuševo, qui revêtaient, en règle générale, un caractère éminemment social. La forme religieuse des interventions était souvent liée à l'échec subi lors des affrontements ouverts avec les autorités : les gens se réfugiaient dans le mysticisme, dans les rêves, réalisant par l'imagination ce qu'ils ne pouvaient pas accomplir dans la vie réelle. $\mathrm{Ne}$ trouvant pas de soutien autour d'eux, ils le cherchaient dans les cieux. Le fait de se tourner vers la religion mordve pouvait aussi, semble-t-il, être lié au souvenir que les habitants de Terjuševo conservaient de leur ancienne liberté, remplacée par le servage et le changement de religion. Il est difficile de dire à quel moment fit son apparition, dans ces rassemblements, un autochtone du nom de Kuz'ma Alekseev. Sans doute prit-il dès le début une part active aux événements. Quoi qu'il en soit, le système de conceptions religieuses et sociales qu'il exposa à ses compatriotes n'a pas pu se présenter immédiatement sous sa forme définitive.

Cet enseignement se résume comme suit : la religion chrétienne est dépassée :

Le Christ n'est plus, et la foi chrétienne va elle aussi cesser d'exister.

5 Il est nécessaire de faire revivre l'ancienne religion mordve, car c'est la seule qui soit authentique. Quand les prières des Mordves parviendront jusqu'à Dieu, douze coups de tonnerre retentiront et depuis le ciel descendront sur la terre David et l'armée des anges, qui jugeront le monde. Après cela, il ne restera plus sur la terre que ceux qui professent la religion mordve, acceptent la loi mordve, adoptent la langue et les vêtements mordves.

Les Mordves seront libres, n'appartiendront plus à des propriétaires et ne payeront plus de redevance. Ils auront la première place, parce que même les maîtres 
porteront des vêtements mordves et seront mordves comme eux. (Zevakin 1936, p. 15).

Toutefois, Kuz'ma Alekseev appelait ses semblables à prier en se tournant non pas vers l'est, comme c'est l'usage dans la religion mordve, mais vers l'ouest, parce que, selon lui, c'est de l'ouest que devait venir la liberté.

7 Selon nous, l'originalité de l'enseignement d'Alekseev reflétait les événements essentiels qui s'étaient produits en Europe et en Russie depuis la fin du XviII siècle. Dans la France révolutionnaire, la religion chrétienne avait commencé à être remplacée par une " religion civile », selon laquelle la force suprême de la Création était la Raison humaine. Les églises furent fermées. Le calendrier chrétien fut aboli. Même le pape, vénéré par les catholiques comme le représentant de Dieu sur la terre, fut emprisonné. Des échos de ces événements parvinrent selon différentes voies jusqu'aux paysans de Mordovie et de nombreux convertis y virent le début de la fin de la religion chrétienne. On n'est pas bien loin de la première assertion de Kuz'ma Alekseev :

Le Christ lui-même a renoncé à son rang, le Christ n'est plus, et la foi chrétienne va cesser d'exister (Zevakin 1936, p. 15).

En 1804, le Premier Consul Napoléon Bonaparte se proclama empereur. Toutefois, les principaux acquis de la révolution ne furent pas remis en question, notamment la liberté des paysans à l'égard des propriétaires fonciers. Partout où passaient les soldats français, le servage était aboli. Dans la guerre qui venait de recommencer contre Napoléon, le gouvernement d'Alexandre I ${ }^{\mathrm{er}}$ essuya une défaite. Après les déroutes de l'armée russe à Austerlitz et à Friedland, le traité de Tilsit, douloureux et humiliant pour la Russie, fut signé en 1807. Les troupes de Napoléon arrivaient aux frontières de l'Empire russe. Les serviteurs de la comtesse de Saint-Priest, des soldats mordves étaient rentrés au pays, racontèrent tout cela comme ils le purent aux gens de Terjuševo. C'est là, selon nous, l'origine de la seconde assertion de Kuz'ma Alekseev, selon laquelle le salut et la liberté des Mordves devaient venir de l'ouest. On ne connaît pas précisément les raisons qui ont conduit le prédicateur à choisir comme messie et sauveur du peuple mordve le roi d'Israël David. Comme on le sait, David, ce jeune homme fragile qui, selon la légende, vainquit en combat singulier le géant Goliath, est souvent considéré par les théologiens comme un symbole de la supériorité de l'esprit sur la force brute et de la victoire des petits sur les grands. C'est peut-être là qu'il convient de chercher l'explication de ce choix. L'origine du caractère fantaisiste de la libération et de la diffusion subséquente de la religion mordve dans le monde entier est probablement à chercher dans la conception religieuse du monde de Kuz'ma Alekseev.

La situation politique intérieure permit de renforcer la lutte de la paysannerie russe pour la liberté sous ses diverses formes, y compris religieuses. Dans le même temps, elle contraignait les propriétaires à surveiller avec la plus grande vigilance le moindre mouvement de ce colosse puissant, mais encore endormi, qui, tout particulièrement dans les districts nationaux, était toujours prêt à se soulever et à se battre quand les conditions le permettaient. Parmi ces régions figuraient depuis des siècles les terres mordves. C'est pourquoi les agissements de Kuz'ma Alekseev ne pouvaient passer longtemps inaperçus. Le 16 septembre 1809, le gouverneur de Nijni-Novgorod, le Conseiller d'État Runovskij, reçut la lettre suivante :

Très honorable Andrej Maksimovič !

Il est de mon devoir de vous informer des circonstances suivantes concernant des rumeurs qui courent dans le district de Nijni-Novgorod et qui sont parvenues jusqu'à moi. Votre Excellence n'est pas sans savoir que le volost' de Terjuševo est 
constitué en grande partie d'une population de convertis d'environ douze mille âmes, qui n'ont pas le droit de tenir des réunions, mais dont certains continuaient en secret à suivre leurs rites primitifs. Voici qu'à présent, sur les terres de Madame Sofia Alekseevna de Saint-Priest, dans le village de Seskin, le paysan Koz'ma, ayant abandonné ses cultures, détourne du droit chemin les convertis des villages environnants et se présente comme un prophète. Mais en quoi consiste sa prophétie, je l'ignore. Il a même déjà osé agir au grand jour en envoyant ses semblables dans les environs de sa localité, notamment dans mes villages de Sivhu, Lom et Inyutinu, dont les starostes et le représentant de la police sont venus m'informer qu'il s'employait à détourner les gens du droit chemin et qu'en son nom ils allaient de maison en maison pour inviter les paysans à une cérémonie religieuse. Dans le village de Seskin, dimanche dernier, c'est-à-dire le 12 septembre, ils ont rassemblé pas moins de quatre mille âmes des deux sexes. Cela pourrait dissimuler un autre danger.

Je prie donc Votre Excellence de bien vouloir prendre en cette occasion les mesures appropriées afin de mettre un terme à tout cela et de diligenter une enquête.

J'ai l'honneur, Votre Excellence, de demeurer éternellement votre très humble serviteur, le Prince Pëtr Trubetzkoj.

Le 14 septembre 1809

au village de Lapšiha » (Zevakin 1936, p. 25)²

Mais n'anticipons pas. Cette lettre du prince Pëtr Sergeevič Trubetzkoj, conseiller d'État ordinaire et membre de l'une des plus illustres familles de la noblesse russe, ne pouvait pas être ignorée, d'autant plus qu'elle concernait les troubles dans le volost' de Terjuševo. Voilà pourquoi dès le lendemain, une instruction secrète du gouverneur fut adressée au chef de la police du zemstvo de Nijni-Novgorod, Sergeev, pour lui demander de procéder à une enquête approfondie sur les événements. Le document lui enjoignait également :

Ensuite, en les tenant sous bonne garde, amener ici le paysan Kuz'ma ainsi que ses principaux complices, afin de les soumettre à de plus amples interrogatoires et de poursuivre l'enquête sur leurs actions illégales. Entre-temps, m'informer sans délai par messager de l'avancement et de la teneur de vos investigations. Exhorter de la façon la plus stricte les paysans à ne pas accorder foi aux fausses prédictions de ce 
prétendu prophète, à conserver leur calme et à se consacrer comme d'habitude à leurs cultures et à leurs besognes, afin de garantir qu'ils ne tiennent aucune réunion secrète ou publique, sous peine des sanctions inévitables prévues par la loi. Confier la charge de surveiller tous leurs mouvements à des policiers non mordves, en leur ordonnant de vous informer immédiatement de tout élément suspect... J'exige que vous m'expliquiez pourquoi vous ne m'avez pas encore signalé un rassemblement d'une telle importance (Zevakin 1936, p. 25-26). peut dire, le gouverneur reçut une lettre de l'archevêque Venjamin de Nijni-Novgorod, ce qui indique que les agents de l'Église étaient également en état d'alerte. Étant donné que cette lettre expose de façon sans doute plus éclairée les principales idées des sermons de Kuz'ma Alekseev, ainsi que leur contexte, j'en cite ici le texte intégralement :

Votre excellence!

Le prêtre Ivan Dmitriev, le 16 septembre, a rapporté à Veniamin ce qui s'était passé en janvier de cette même année : le néophyte Kozma Alekseev répand parmi les siens ses paroles «tentatrices", selon lesquelles bientôt leur ancienne foi mordve « s'élèverait » et que la religion chrétienne déclinerait.

Ces bruits ont été tellement insistants que les autorités se sont emparées de ce néophyte et l'ont emmené dans le village de Lyskogo chez le prince Egor Gruzinskij qui règne sur ce territoire; il est resté là jusqu'aux saintes Pâques; puis, le second jour des sept ?, il est apparu dans le village de Sarlej; il a assisté longuement aux fêtes à l'église, mieux que par le passé, et déclaré que deux fois, à Lyskovo, il s'était confessé et avait communié aux "saints mystères ». Trois semaines plus tard, les autorités du volost' ont envoyé à l'église une copie avec ordre de Son Excellence où il est écrit entre autres que la direction dudit volost' doit surveiller ce néophyte et, au premier cas de récidive, adresser un rapport à Son Excellence et placer ledit néophyte sous bonne garde, l'exiler et l'assigner à résidence. À la mi-mai, le néophyte de nouveau usa de sa parole tentatrice, raison pour laquelle les derniers jours de mai il fut arrêté et mené à Son Excellence le prince Gruzinskij pour y rester jusqu'à ce mois de septembre.

À présent, de retour chez lui, il «tente " de nouveau les siens : ils se groupent en nombre autour de lui, recréent dans les champs selon leur vieille coutume mordve des lieux de culte qu'ils vénéraient en secret, à la suite de quoi samedi dernier, à l'approche du soir, on a vu des néophytes se rendre en masse auprès d'Alekseev, et toute cette assemblée s'est prolongée la nuit et le dimanche matin. Après la liturgie dominicale, le prêtre Ioann ainsi que son compagnon ont gagné une éminence et vu à proximité des bois, non loin du village de Seskin où vit le tentateur, une foule abondante et des feux en grand nombre où brûlaient manifestement des restes d'animaux. Ceux qui s'y rendaient leur dirent sans peur qu'ils allaient au temple païen retrouver un prophète, et au retour racontaient que ce prophète est l'esprit du nom de Melčeje. On raconte aussi que, d'après ce faux prophète, le Christ, c'est un rang. Le Christ étant vieux, il s'est retiré. Il n'est plus de Christ, il n'y aura plus de foi chrétienne. Ce rang est conféré à un autre. Celui-ci viendra de l'ouest, c'est pourquoi nous devons prier vers l'ouest. On entend aussi qu'il y aura bientôt un Jugement terrible à l'endroit même où ils célèbrent leur culte païen. C'est pourquoi quelques-uns des néophytes qui y croient partagent leur bien, tandis que d'autres ne plantent ni blé, ni grain.

J'ai l'honneur de vous demander, gouverneur miséricordieux, de soumettre à surveillance les actes de néophytes tels que Kozma et ses disciples. Pour ce faire et pour exhorter le paysan mentionné comme les autres (brebis) égarées, il est indispensable de dépêcher dans le village de Bol'še Seskino un prêtre qui saura y faire. Dans ce cas, il se rendra là-bas avec les recommandations adéquates de ma part.

Avec mon sincère respect et zèle envers votre Excellence, gouverneur 
miséricordieux,

Votre fidèle serviteur et pèlerin, A.Veniamin (Zevakin 1936, p. 26-28) sacrificiels et de boissons rituelles (pure), autres attributs de l'ancienne religion mordve, les habitants de Terjuševo utilisaient dans leurs prières des icônes chrétiennes, par exemple celle de Saint-Nicolas thaumaturge. Certes, il n'est pas impossible que ces icônes aient été utilisées à des fins de "conspiration». Comme on peut le constater d'après les dénonciations de Trubetzkoj, de Veniamin, et même d'après l'ordre secret de Runovskij, les autorités provinciales étaient préoccupées non pas tant par l'aspect religieux des sermons d'Alekseev que par la protestation sociale qu'ils véhiculaient, le désir de libération du peuple mordve, le grand nombre de ses partisans, et surtout le caractère public de leurs réunions. L'absence de crainte de la part des paysans, c'était là ce que leurs oppresseurs redoutaient le plus. La machine judiciaire impériale se mit au travail. Kuz'ma Alekseev et sept de ses principaux partisans - Nikita Ivanov, 45 ans, Mihail Frolov, 45 ans, Pëtr Maksimov, 43 ans, Nikolaj Alekseev, 26 ans, Boris Ivanov, 27 ans, Jakim Ivanov, 30 ans, Filipp Savel'ev, 30 ans - furent arrêtés. Lors de l'arrestation de Kuz'ma, une foule d'habitants de son village se rassembla dans la cour, prête à lui venir en aide. Pourtant, non seulement il ne demanda par d'aide, mais il fit tout ce qu'il put pour rassurer ses partisans, en leur disant que son affaire ne les concernait pas. Il les exhorta à ne pas oublier la foi mordve, à prier le vendredi et le dimanche et à attendre la liberté et le salut qui ne manqueraient pas d'arriver de l'ouest. Compte tenu de l'état d'esprit des paysans, on peut dire que cette attitude d'Alekseev est la seule chose qui permit d'éviter un nouveau soulèvement à Terjuševo.

Le 30 septembre, le gouverneur Runovskij proposa au tribunal de l'uezd de NijniNovgorod d'examiner l'affaire "sans le moindre retard ». Le même jour, il informa en détail le ministre de l'Intérieur Aleksej Kurakin, ajoutant à titre personnel que la cause des agissements de Kuz'ma Alekseev était manifestement le désir de celui-ci de recevoir des présents de la part des paysans. Le gouverneur assurait le ministre du caractère insignifiant de l'incident. Toutefois, le ministre et le gouvernement prirent l'affaire très au sérieux. À la mi-octobre, des prêtres et des fonctionnaires furent envoyés dans tous les villages mordves du volost' de Terjuševo, afin d'exhorter et de menacer les habitants. Les villages de Kurilovo, Borisovo, Tepelevo, Sarlej, Surovatihu, Teploe et Armanihu furent honorés par la visite de l'archevêque Veniamin de Nijni-Novgorod et d'Arzamas. La population paysanne de tous les villages mordves du kraï fut divisée en groupes de dix à quinze fermes, dans chacun duquel fut désigné un responsable d'origine mordve. Dans le cas où des paysans participaient en secret à des prières mordves ou à des réunions, ces responsables avaient l'obligation d'en informer la direction du domaine et $\mathrm{du}$ volost', faute de quoi eux-mêmes ou leurs fils pourraient être incorporés dans l'armée. Seuls les Russes étaient autorisés à avoir des fonctions dans l'administration $\mathrm{du}$ volost' et du domaine.

Le 8 novembre, le gouverneur de Nijni-Novgorod reçut un message du ministère de l'Intérieur qui disait :

J'ai l'honneur d'informer Votre Excellence que j'ai rendu compte à Sa Majesté Impériale de votre rapport concernant le faux prophète apparu dans la province qui vous est confiée, le paysan Alekseev, et que Sa Majesté a bien voulu honorer de sa Haute approbation les mesures adoptées concernant les convertis qui sont dans l'erreur.

Original signé par le ministre de l'Intérieur Aleksej Kurakin.

Vérifié sur l'original par le conseiller titulaire Alekseev (Zevakin 1936, p. 52).

Études finno-ougriennes, 47 | 2015 
La lettre suivante adressée au gouverneur lui ordonne d'informer en détail le ministère de la décision judiciaire rendue dans cette affaire. Le 6 décembre 1809, le gouverneur proposa au Tribunal pénal de la province de Nijni-Novgorod d'examiner celle-ci dans les meilleurs délais :

Son Excellence le ministre de l'Intérieur, le prince Aleksej Borisovič Kurakin, sur la base de mon rapport relatif aux nouvelles instructions des autorités civiles et religieuses visant à empêcher les convertis d'origine mordve de tomber dans l'erreur où tentait de les attirer par cupidité le paysan Alekseev, me demande de lui rendre compte en temps voulu de la décision qui sera rendue par le tribunal concernant le paysan Alekseev susmentionné ainsi que ses complices. C'est pourquoi j'invite le Tribunal pénal à rendre dans les meilleurs délais sa décision dans cette affaire et à m'en communiquer copie (Zevakin 1936, p. 54).

Les activités de Kuz'ma Alekseev n'étaient évidemment motivées par aucune « cupidité ». Par ce mensonge, Runovskij suggère clairement au tribunal des arguments pour la sentence. De façon générale, en vertu des lois de l'Empire russe, les entorses à la religion chrétienne devaient être jugées par un tribunal ecclésiastique. Mais celui-ci ne pouvait pas condamner quelqu'un à l'exil et encore moins à la peine capitale. L'affaire d'Alekseev pouvait être portée devant une juridiction pénale en cas d'appel au renversement du gouvernement en place, d'un appel à la violence pour se libérer des propriétaires ou de rébellion. Or il n'y avait rien de tel dans ses activités. Il appelait simplement à restaurer la religion mordve et à attendre la liberté. Ainsi, même en vertu des lois draconiennes de l'Empire russe, un procès pénal contre Kuz'ma Alekseev avait un caractère arbitraire. Toutefois, cela ne dérangeait nullement Runovskij, ni Kurakin, ni le tsar Alexandre. Le tribunal pénal de Nijni-Novgorod se saisit de l'affaire avec zèle et empressement.

Comment expliquer tout cela?

Le prophète mordve ne disait rien de nouveau, rien qui se distingue des nombreuses autres idées qui, à l'époque de Kuz'ma et très longtemps après lui, circulaient dans tous les coins du monde mordve

soulignait le célèbre chercheur prérévolutionnaire, spécialiste des Mordves, N. I. Smirnov. Selon lui, la répression fut causée par le fait que

les discours du Saint-Jean mordve avaient des accents sociaux qui ont alarmé

l'administration et les propriétaires fonciers locaux (Smirnov 1895, p. 104).

Il est probable que c'est là la raison qui poussa les autorités à enfreindre les lois qu'elles avaient elles-mêmes établies.

Selon le dossier de l'affaire, Kuz'ma Alekseev naquit et fut baptisé à la fin d'octobre 1764. Lors du prononcé du jugement, il avait 45 ans révolus. Jusqu'en 1802, il cultiva la terre. Puis, pendant sept ans, il fabriqua du charbon dans la forêt. Depuis l'enfance, en plus des rites chrétiens, il pratiquait également les anciens rites mordves. Chez lui étaient constamment présentes et s'affrontaient deux religions, deux ensembles de prières: les prières chrétiennes, qu'il disait en russe, et les prières mordves, qu'il disait dans sa langue maternelle. Ses sept années de vie dans la forêt, sans contact fréquent avec les prêtres et la population russophone, conduisirent à rétablir dans son âme les bases de l'ancienne religion mordve. Peut-être celle-ci, à son tour, stimula-t-elle le développement de sa conscience identitaire. Une fois revenu chez lui, dans son village, il dit avoir commencé à entendre une voix qui lui révélait les canons de la « vraie foi » et les moyens d'agir.

Ses sept disciples qui furent traduits en justice montrèrent qu'ils n'avaient pas 
[...] renié la loi chrétienne, qu'ils allaient à l'église et accomplissaient tous les rites chrétiens. Mais, ayant été formés par leurs pères, ils ne renonçaient pas à leurs anciens rites mordves, qu'ils pratiquaient depuis leur enfance (Zevakin 1936, p. 57)

À la question de savoir si Kuz'ma Alekseev avait entendu une « voix » et s'ils pensaient que ses sermons étaient la vérité, six accusés répondirent qu'ils ne savaient pas s'il avait ou non entendu ces voix, mais qu'Alekseev était un homme modéré et qu'il ne proposait aux gens rien d'autre que de prier Dieu selon le rite mordve. Ils considéraient cette proposition comme juste et ne pouvaient plus désormais renoncer à ces rites. L'accusé Nikolaj Alekseev ajouta à cela qu'il considérait toutes les prophéties de Kuz'ma

[...] aujourd'hui encore comme vraies, et pour lui, Alekseev, ces voix qu'il déclarait entendre, pouvaient fort bien exister, parce que lui, Alekseev, était un homme modéré et qu'il suivait toujours le rite mordve quand il priait. Il était donc digne de la révélation qu'il transmettait et lui, Alekseev (cette fois-ci, Nikolaj - V.A.), ne pouvait pas renoncer aux rites mordves (Zevakin 1936, p. 61).

21 Les déclarations des huit accusés concernant leur attachement à la religion et aux coutumes mordves facilitèrent grandement la tâche des juristes complaisants. Au terme d'une brève délibération, ils parvinrent à une conclusion « mûrement réfléchie » qui coïncidait dans les grandes lignes avec l'avis du gouverneur sur les activités de Kuz'ma Alekseev :

Qu'il exposait aux paysans des révélations absurdes de sa propre invention. Mais les raisons qui le poussaient à faire tout cela pouvaient être diverses : il se pouvait que ce fût avec l'intention de nuire, pour détourner les gens du droit chemin et tirer profit de leurs biens, ou alors voulait-il les raffermir dans ces rites par son attachement irrationnel, par sa fausse piété et par ses convictions concernant la préservation et la diffusion de ladite pratique mordve. Mais même si tout cela s'est produit du fait d'un attachement absurde aux rites mordves, tout ce qu'il a fait est impardonnable, dans la mesure où il a installé chez les paysans une grande dépravation et un relâchement dans la religion chrétienne. En conséquence de quoi Alekseev, pour cette révélation absurde inventée par lui, ainsi que pour l'organisation, en présence de nombreuses personnes, de cérémonies religieuses factices selon le rite mordve et contraires à la religion chrétienne, est condamné, conformément à l'article $254 \mathrm{du}$ Statut de la circonscription ecclésiastique et à l'article 202 du Code militaire, en proportion de ses actions, à recevoir 80 coups de fouet dans le village de Bolšoe Seskino, en présence des Mordves convertis ayant participé et réunis en prière, ensuite de quoi, afin d'éviter qu'il continue à dépraver les paysans par lui, il sera déporté dans la province d'Irkoutsk (Zevakin 1936, p. 63-64).

Concernant les autres accusés, l'arrêt poursuit :

Nikita et Boris Ivanov, paysans du village de Bol'šoe Seskino, croyant aveuglément l'injonction qu'il leur avait faite, allèrent de village en village en propageant ses idées, et en appelant les paysans de tout le volost' à une prière collective ; ... de plus Mihail Frolov, Pëtr Maksimov, Nikolaj Alekseev, Jakov Ivanov et Filip Savel'ev du village de Kužutok assistèrent ledit Alekseev pour la prière du 12 septembre selon leur ancienne coutume mordve. C'est pourquoi, pour cette participation, à la mesure de la faute de chacun, sur la base de l'article $129 \mathrm{du}$ code militaire, ils seront condamnés à 40 coups de fouet, et les deux premiers, Boris et Nikita Ivanov - parce que plus que les autres, ils ont participé à cette supplique paysanne -, les enrôler dans l'armée, avec l'accord de leur propriétaire, et en cas d'incapacité pour le service, ils seront déportés dans cette même province d'Irkoutsk (Zevakin 1936, p. 64).

L'arrêt poursuit :

Lors de l'exécution du châtiment sur les condamnés, interdire formellement à tous les convertis mordves du volost' de Terjusevo de tenir à l'avenir des 
rassemblements destinés à effectuer des cérémonies d'après les anciens usages mordves, tant ouvertement qu'en secret, sous peine d'un jugement sévère conformément aux lois. Veiller à cet égard à maintenir une surveillance attentive du tribunal du zemstvo.

Le Président Karl Rebinder, le conseiller David Čekerljan, les assesseurs de la noblesse Sergej Skuridin, Evgraf Babkin, l'assesseur des marchands Sergej Pačkunov. Confirmé par le secrétaire Sergej Il'in. Signé le 11 janvier 1810. Lu par l'avoué régional des affaires pénales le 12 janvier...

Conformité avec l'original vérifiée par l'employé de bureau Aleksandr Evenius (Zevakin 1936, p. 64-65).

Dans une lettre au ministre Kurakin, le gouverneur Runovskij présenta ce jugement. Le 19 mars, il reçut une réponse qui disait :

Le rapport de Votre Excellence en date du 15 février, comportant un extrait de la décision du Tribunal pénal de Nijni-Novgorod concernant le paysan Kuz'ma Alekseev et ses complices, condamnés pour avoir divulgué de fausses rumeurs visant à débaucher les convertis mordves et à affaiblir chez eux la religion chrétienne, a été porté à l'attention de Sa Majesté Impériale qui a daigné ordonner ce qui suit.

Le verdict du Tribunal pénal relatif aux paysans susmentionnés doit être mis à exécution, à l'exclusion des châtiments corporels dont tous doivent être dispensés.

J'ai l'honneur de communiquer cette volonté suprême à votre Excellence en vue de la bonne exécution de celle-ci.

Le Ministre de l'Intérieur

signé sur l'original par le prince Aleksej Kurakin (Zevakin 1936, p. 67).

Cependant, la " grâce » impériale ne put épargner le fouet qu'à Nikolaj Alekseev. Pour les autres, la peine fut exécutée, et les condamnés à ce moment-là étaient déjà en route pour la Sibérie. Après la présentation de plusieurs documents justifiant la nonexécution de l'« ordre impérial », à la fin de juillet 1810, l'affaire fut clôturée.

Malgré l'importance de la dimension sociale dans les activités de Kuz'ma Alekseev, on ne doit pas cependant oublier son enseignement religieux. Sur ce plan, il se présente comme un réformateur de l'ancienne religion mordve, dans laquelle il a incorporé des éléments juifs (le roi David) et chrétiens (Saint-Nicolas). Dans les sermons d'Alekseev, nous rencontrons le passage définitif du polythéisme - qui comportait certes un dieu suprême, Shkaem - au monothéisme.

Les prières sont faites non seulement le vendredi, ce qui était le cas chez les Mordves probablement depuis l'époque de la Horde d'or, mais aussi le dimanche, ce qui est évidemment dû à l'influence chrétienne. Une nouveauté radicale est l'obligation de prier en se tournant non vers l'est (lever du soleil), mais vers l'ouest. Les raisons possibles à cela ont été discutées plus haut. Et les officiants de la prière (appelés ozatja et puren'atja) ne sont pas élus, comme c'était l'usage, mais nommés par Kuz'ma luimême. Tout l'enseignement d'Alekseev est imprégné d'un fort sentiment identitaire : tout ce qui est mordve est lié pour lui à la religion mordve. Si l'on considère que l'arrivée du christianisme s'est accompagnée par l'assimilation de la culture mordve et de tout le peuple mordve, le désir de l'opposer à la religion mordve peut être considéré comme une tentative pour stimuler la conscience identitaire du peuple, pour l'unir dans la lutte pour la survie. La nouvelle religion mordve dans laquelle Alekseev, dans ces conditions, devait voir la seule force capable de sauver les Mordves en tant que peuple distinct, lui apparaissait non pas comme le vieux panthéon des dieux païens, mais comme un ensemble formé sur la base de l'ancienne religion mordve, du judaïsme et du christianisme. Il semble avoir essayé de prendre le meilleur de chaque religion. 
Pour ce paysan illettré, il était extraordinairement difficile de conduire ses gens non pas à se battre, ni à piller les demeures des propriétaires, ni même à résister aux expéditions punitives, mais à entrer dans le nouveau monde des sentiments et des idées de la religion. Bien sûr, Kuz'ma Aleekseev fut tout d'abord aidé par les aspirations et les espoirs des paysans eux-mêmes. Ses qualités personnelles - l'intelligence, la probité, la foi profonde dans son destin personnel ainsi que son exceptionnel talent oratoire jouèrent également un rôle important.

La figure hors du commun du " prophète mordve » a retenu l'attention des chercheurs prérévolutionnaires et soviétiques, parmi lesquels V. I. Sneževskij, qui écrivit en 1892 dans le $n^{\circ} 10$ de la revue Istoričeskij vestnik (Le Bulletin historique) un remarquable article intitulé « Kuz'ma, le prophète mordve de Terjuševo "; N. I. Smirnov, qui compila notamment la première biographie relative au personnage; T.V.Vasil'ev, qui lui consacra de nombreuses lignes dans son livre "La Mordovie", publié en 1931; M. I. Zevakin, qui publia sur Kuz'ma Alekseev toute une monographie, etc. Malheureusement, il existe aussi une littérature d'un autre type, visant à déformer l'image de cet homme pur et à lui imposer, en quelque sorte, un bagne moral. Après son procès, il a été la cible, dans un certain nombre de journaux, de calomnies et de moqueries. En 1866, le magazine Otečestvennye zapiski (Mémoires patriotiques), dans ses numéros d'août et de septembre, n'eut aucun scrupule à publier à son sujet un article plein de sous-entendus et d'insultes. Plus tard fut publiée une brochure séparée au contenu similaire, dont on trouve aujourd'hui encore des copies manuscrites ici ou là.

Ces insinuations reçurent fort heureusement des réponses adéquates de la part des chercheurs russes sérieux. C'est ainsi que N.I.Smirnov, dans la bibliographie commentée de sa monographie Mordva (Les Mordves), écrit au sujet du pamphlet publié dans les Otečestvennye zapiski :

L'auteur s'écarte du terrain de la factualité scientifique et s'engage dans le domaine de l'exercice littéraire à partir d'un canevas constitué de fragments déformés empruntés à la tradition populaire (russe - V.A.). K. nous donne une description détaillée de l'apparence du prophète mordve et lui prête des aventures que nul ne pouvait connaître... Ces développements littéraires de médiocre qualité occupent la totalité de l'article... (Smirnov 1895, p. 266)

De tels écrits, qui étaient devenus un phénomène nouveau dans le combat contre les partisans de la liberté et la dignité de leur peuple, avaient pour but d'altérer leur image positive voire de les effacer de la mémoire des générations futures.

Mais Kuz'ma Alekseev ne fut pas oublié par ses compatriotes. Les Mordves de Terjuševo continuèrent encore longtemps à se rendre à des réunions secrètes et à prier comme il le leur avait enseigné.

Ils accomplissaient leurs rites selon leur manière ancienne, écrit Smirnov, mais ils ne leur associaient plus aucune attente. (Smirnov 1895, p. 105)

Les événements des années 1804-1810 furent le dernier grand mouvement identitaire des Mordves de Terjuševo. Leurs ancêtres s'étaient battus vaillamment sous la bannière de Purgaz $^{3}$ et d'Alabuga ${ }^{4}$. Ils s'étaient lancés à l'assaut des forteresses tsaristes avec Moskov et Vorkadin'. Ils avaient pillé les domaines fonciers dans les unités d'Akaj Boljaev ${ }^{6}$, d'Aljona d'Arzamas ${ }^{7}$ et de Nesmejan Vasil'ev8.

30 Il ne fait aucun doute que les Mordves de Terjuševo, installés dans les alentours de Nijni-Novgorod - principale base de la politique coloniale tsariste dans la région de la Volga -, qui furent toujours les premiers à recevoir les coups de l'appareil administratif 
et ecclésiastique, par leur combat long et acharné pour préserver leur indépendance, leur culture, leur religion et leur identité, ont non seulement sauvé de l'assimilation une partie importante de la région mordve, mais aussi affaibli la pression du tsarisme sur les autres peuples non russes. Cette lutte séculaire contre le puissant appareil étatique et ecclésiastique d'un immense empire a épuisé peu à peu les forces des Mordves de Terjuševo, aussi tenace que fût cette population. Actuellement, leurs villages russifiés sont situés en majeure partie dans le raïon de Dal'ne Konstantinovo de l'oblast de Nijni-Novgorod. Ils ont perdu leur langue, leur identité, et seules les particularités de leur culture et de leur psychologie rappellent encore leur héroïque passé mordve.

\section{BIBLIOGRAPHIE}

LÉNINE Vladimir 1968 = ЛЕНИН ВЛАДИМИР ИЛЬИЧ, « ПАМЯТИ ГЕРЦЕНА » (Souvenir de Herzen), полн. СоБР. СОЧ. (5 Изд.) (Euvres complètes $5^{\mathrm{e}}$ édition). т. 21. М.: полИТИЗДАТ.

SMIRNOV Ivan 1895 = СМИРНОВ ИВАН НИКОЛАЕВИЧ, МОРДВА (Les Mordves), КАЗАНЬ.

VASIL'EV Andrej 1965 = ВАСИЛЬЕВ АНДРЕЙ, С. П. ТРУБЕЦКОЙ (S.P. Trubetzkoï), Л.

ZEVAKIN M. 1936 = ЗЕВАКИН М. И., «КУЗЬМА АЛЕКСЕЕВ» (Kuz'ma Alekseev), КРЕСТЬЯНСКОЕ

ДВИЖЕНИЕ МОРДВЫ ТЕРЮШЕВСКОЙ ВОЛОСТИ (1808-1810 ГГ.): МАТЕРИАЛЫ АРХИВА (Le mouvement paysan des Mordves de la volost de Terjuševo), САРАНСК.

\section{NOTES}

1. Poète, descendant de la famille impériale d'Iméréthie, Arčil II occupa à plusieurs reprises le trône impérial entre 1661 et 1698. Il en fut chassé du fait d'une intervention turque et dut se réfugier à Moscou, où il avait déjà fait un séjour de plusieurs années. Il y mourut en exil et fonda la communauté géorgienne de Moscou (Ndlr).

2. Ici et par la suite, la version originale de ces documents est présentée sans rectifications $(\mathrm{NdA})$.

3. Purgaz ou Purgas, prince erza du XIII ${ }^{\mathrm{e}}$ siècle, mentionné dans les chroniques russes, dirigeant d'une entité appelée volost de Purgas (Ndlr).

4. Alabuga, prince mordve du XIve siècle, mentionné dans les chroniques russes, combat du côté de la Horde contre les Russes et fédère les tribus mordves septentrionales sous sa houlette (Ndlr).

5. Vorkadin Činkov (erza), avec Moskov, ont dirigé en 1606-1607, à l'époque des troubles, une insurrection dans la région de Nijni-Novgorod (Ndlr).

6. Akaj Boljaev, chef (murza) mordve, leader de l'insurrection paysanne de 1670-1671 (Ndlr). 
7. Aljona d'Arzamas, religieuse née dans une famille cosaque, leader d'un régiment de paysans insurgés lors de l'insurrection de Stepan Razin en 1670-1671 (Ndlr).

8. Nesmejan Vasiljev, leader de l'insurrection mordve de Terjuševo en 1743-45 (Ndlr).

\section{RÉSUMÉS}

Cet article relate le mouvement religieux et national d'un groupe ethnographique mordve, les Terjuh, vivant dans la région de la Volga. Ce mouvement s'est déroulé dans les années 1808-1810 et a été dirigé par Kuz'ma Alekseev. Ce groupe était la seule partie de la population mordve qui se trouvait soumise au droit féodal et qui, dans le kraï de Nižegorod, mena un combat résolu pour sa liberté. Ce mouvement des Terjuh pour se libérer du servage reflète de manière curieuse les idées de la Révolution française de 1789. Celles-ci se combinaient avec les idées d'une renaissance nationale des Mordves, que Kuz'ma Alekseev voyait sous forme d'une nouvelle religion ethnique, fondée sur un ensemble combinant des orientations issues des religions mordve, judaïque et chrétienne. Ce mouvement ne toucha qu'une volost et quelques milliers de paysans mordves; il n'en reste pas moins qu'il fit peur au gouvernement de la Russie, de sorte que c'est l'empereur Alexandre $1^{\mathrm{er}}$ en personne qui dirigea son écrasement.

This article presents an ethnic religious movement in a Mordvin ethnic group, the Teryukh, living in the Volga region. It took place in 1808-1810 and was led by Kuzma Alekseev. This group was the only part of the Mordvin population that was under feudal rule and that fought fiercely for freedom in the Nizhegorod region. This Teryukh movement in order to emancipate themselves from serfdom curiously reflects ideas from the 1789 French revolution, combined with ideas of Mordvin revival under the form of a new ethnic religion. Kuzma Alekseev saw this new religion as a mix of elements from the Mordvin, Judaic and Christian religions. This movement concerned only one volost and some thousands of Mordvin peasants; still, it frightened the Russian government so deeply that its repression was led personally by tsar Alexandre I.

В СТАТЬЕ ОПИСЫВАЕТСЯ НАЦИОНАЛЬНО-РЕЛИГИОЗНОЕ ДВИЖЕНИЕ МОРДОВСКОЙ ЭТНОГРАФИЧЕСКОЙ ГРУППЫ - ТЕРЮХАН, ЖИВУЩИХ В ПОВОЛЖЬЕ, В 1808-1810 ГГ., ПОД РУКОВОДСТВОМ КРЕСТЬЯНИНА КУЗЬМЫ АЛЕКСЕЕВА. ЭТА ГРУППА БЫЛА ЕДИНСТВЕННОЙ ЧАСТЬЮ МОРДОВСКОГО НАСЕЛЕНИЯ, НАХОДЯЩЕЙСЯ В КРЕПОСТНОЙ ЗАВИСИМОСТИ И ВЫДЕЛЯЛАСЬ В НИЖЕГОРОДСКОМ КРАЕ ОСОБО УПОРНОЙ БОРЬБОЙ ЗА СВОБОДУ. В ДВИЖЕНИИ ТЕРЮХАН ЗА ОСВОБОЖДЕНИЕ ОТ КРЕПОСТНИЧЕСТВА В СВОЕОБРАЗНОЙ ФОРМЕ НАШЛИ ОТРАЖЕНИЕ ИДЕИ ВЕЛИКОЙ ФРАНЦУЗСКОЙ РЕВОЛЮЦИИ 1789 Г. ОНИ СОЧЕТАЛИСЬ С ИДЕЯМИ НАЦИОНАЛЬНОГО ВОЗРОЖДЕНИЯ МОРДВЫ, КОТОРОЕ КУЗЬМА АЛЕКСЕЕВ ВИДЕЛ В СОЗДАНИИ НОВОЙ НАЦИОНАЛЬНОЙ РЕЛИГИИ, ОСНОВАННОЙ НА КОМПЛЕКСЕ УСТАНОВОК ДРЕВНЕЙ МОРДОВСКОЙ, ИУДЕЙСКОЙ И ХРИСТИАНСКОЙ РЕЛИГИЙ. ХОТЯ ЭТО ДВИЖЕНИЕ НЕ ВЫШЛО ЗА ПРЕДЕЛЫ ОДНОЙ ЛИШЬ ВОЛОСТИ И В НЕМ УЧАСТВОВАЛО ВСЕГО ЛИШЬ НЕСКОЛЬКО ТЫСЯЧ МОРДОВСКИХ КРЕСТЬЯН, ОНО НАСТОЛЬКО НАПУГАЛО ПРАВИТЕЛЬСТВО РОССИИ, ЧТО ЕГО ПОДАВЛЕНИЕМ РУКОВОДИЛ ЛИЧНО ИМПЕРАТОР АЛЕКСАНДР 1. 


\section{INDEX}

Keywords : Mordvinian Religion, New Religion, Peasant Movement, Repression, Religious Revitalisation, Uprising

Index chronologique : XIXe siècle (début), XVIe siècle

motscleset mordva religioon, repressioon, talupoja liikumine, usuline revitalisatsioon, uus religioon, ülestõus

Index géographique : Armanihu, Arzamas, Austerlitz, Bol'še Seskino, Borisovo, Dal'ne Konstantinovo, Fédération de Russie, Friedland, Inyutinu, Irkoutsk (oblast'), Kurilovo, Lom, Lyskogo, Nižni Novgorod (oblast'), Sarlej, Seskin, Sivhu, Surovatihu, Tepelevo, Teploe, Terjuševo, Tilsit, Volga (vallée de la), Alabuga

Mots-clés : Iméréthie, Décembristes, mouvement paysan, nouvelle religion, revitalisation religieuse, religion mordve, répression, soulèvement

motsclesru КРЕСТЬЯНСКОЕ ДВИЖЕНИЕ, МОРДОВСКАЯ РЕЛИГИЯ, РЕПРЕССИИ, РЕЛИГИОЗНАЯ РЕВИТАЛИЗАЦИЯ, ВОССТЯНИЕ

nomsmotscles Mordves, Perses, Turcs, Russes

Thèmes : histoire 